\title{
Distribution of EFHC1 or Myoclonin 1 in mouse neural structures
}

\section{Christine Léon ${ }^{a}$, Laurence de Nijs ${ }^{a}$, Grazyna Chanas a , Antonio V. Delgado-Escuetab $^{b}$, Thierry Grisar ${ }^{a, *}$, Bernard Lakaye ${ }^{a}$}

\author{
a GIGA-Neurosciences, University of Liège, Avenue de l'Hopital 1, Liège, Belgium \\ ${ }^{\mathrm{b}}$ Epilepsy Genetics/Genomics Laboratories, Comprehensive Epilepsy Program, David Geffen School of Medicine at UCLA and VA \\ GLAHS-West Los Angeles Medical Center, Los Angeles, CA 90073, USA
}

Received 28 August 2009; received in revised form 16 October 2009; accepted 15 November 2009 Available online 16 December 2009

\author{
KEYWORDS \\ Juvenile myoclonic \\ epilepsy; \\ Brain development; \\ Ependymal cells; \\ Radial glia; \\ Motile cilia; \\ Neural stem cells
}

\begin{abstract}
Summary EFHC1, a gene mutated in juvenile myoclonic epilepsy, encodes EFHC1, a protein with three DM10 domains and one EF-hand motif. We recently demonstrated that this molecule is a microtubule-associated protein (MAP) implicated in neuronal migration. Because some controversies persist about the precise localization in the CNS, we studied the neuroanatomical distribution of EFHC1 in mature and developing mouse brain. In the adult, low mRNA expression was detected in several brain structures such as cortex, striatum, hippocampus and cerebellum. At E16, EFHC1 mRNA was shown to be expressed in cortex and not only in cells lining ventricles.

Using a purified polyclonal antibody, EFHC1 staining was observed in all cortical layers, in piriform cortex, in hippocampus and in Purkinje cells of cerebellum. In the cortex, the majority of EFHC1 positive cells correspond to neurons, however some glial cells were also stained. In agreement with a previous study, we demonstrated strong EFHC1 expression in cilia of ependymal cells lining cerebral ventricles. Moreover, at E16, the protein was observed at the borders of brain ventricles, in choroid plexus, but also, although to a lesser extent, in piriform and neocortex. In these latter structures, the pattern of expression seems to correspond to the extensions of the radial glia fibers as demonstrated by BLBP immunostaining. Finally, we confirmed that EFHC1 was also expressed and co-localized with the mitotic spindle of neural stem cells.

These results confirm that EFHC1 is a protein with a likely low expression level in mouse brain but detectable both in adult and embryonic brain supporting our previous data and
\end{abstract}

Abbreviations: ABC, avidin-biotin-peroxidase complex; BLBP, brain lipid-binding protein; DAB3, 3'-diaminobenzidine; FITC, fluorescein isothiocyanate; GFAP, glial fibrillary acidic protein; IgG, immunoglobulin G; JME, juvenile myoclonic epilepsy; MAP, microtubule-associated protein; MTOC, microtubule-organizing center; NeuN, neuronal nuclei; NGS, normal goat serum; NSC, neural stem cells; PBS, phosphatebuffered saline; PBSG, phosphate-buffered saline with $4.5 \mathrm{~g} /$ l glucose; PFA, paraformaldehyde; RRX, rhodamine Red-X; VDCC, voltagedependent calcium channel.

* Corresponding author. Tel.: +32 36659 54; fax: +32 3665953.

E-mail address: tgrisar@ulg.ac.be (T. Grisar). 
hypothesis that EFHC1 could play an important role during brain development. As discussed, this opens the door to a new conceptual approach viewing some idiopathic generalized epilepsies as developmental diseases instead of classical channelopathies.

(c) 2009 Elsevier B.V. All rights reserved.

\section{Introduction}

Juvenile myoclonic epilepsy (JME) is the most frequent form of idiopathic generalized epilepsy. It accounts for 5-10\% of all epilepsies and appears between 8 and 26 years. JME involves short involuntary muscle contractions, tonic-clonic seizures and sometimes absences (Wolf, 1992).

In 2004, Suzuki et al. identified, in different unrelated families with JME probants, several missense mutations in a gene called EFHC1. Since then, several new mutations have been identified in various populations and it seems that $9 \%$ of JME cases in Mexico and Honduras and 3\% of clinic patients from Japan carry mutations in EFHC1. This represents the highest number and percentage of mutations found for $a$ juvenile myoclonic epilepsy causing gene of any population group (Medina et al., 2008). The gene encodes a 75-kDa protein with three DM10 domains of unknown function and a single EF-hand motif, a $\mathrm{Ca}^{2+}$-binding domain. The transcript was shown to be expressed in many human tissues, including brain, also highest levels were detected in lung and testis (Suzuki et al., 2004). It was first proposed that EFHC1 was "pro-apoptotic"' as its overexpression in hippocampal neurons in vitro induced apoptotic cell death. This effect was significantly reduced by any of the five mutations associated with JME. Patch-clamp analysis of BHK (Baby Hamster Kidney) cells transfected with $\mathrm{Ca}_{\mathrm{v}} 2.3$ VDCC (voltage-dependent calcium channel) and EFHC1 showed significantly increased R-type $\mathrm{Ca}^{2+}$ currents. So, the pro-apoptotic effect of EFHC1 was assigned to this enhancing effect on $\mathrm{Ca}^{2+}$ through $\mathrm{Ca}_{\mathrm{v}} 2.3$ VDCC (Suzuki et al., 2004).

In 2005, another research group pointed out that EFHC1 is orthologous to Rib72, an axonemal protein of Chlamydomonas reinhardtii. They demonstrated that EFHC1 is abundantly expressed in mouse tissues that have motile cilia or flagella including the brain and suggested that it plays a role in the intrinsic properties of these organelles (Ikeda et al., 2005).

Our laboratory previously reported that the subcellular distribution of EFHC1 in different cell lines varied during the cell cycle. In interphase cells, the protein is present in the cytoplasm and nucleus, except nucleoli and is particularly concentrated at the centrosome. During mitosis, EFHC1 is localized at spindle poles of the mitotic spindle and also at the midbody during cytokinesis (de Nijs et al., 2006). These results suggest that EFHC1 could play an important role during cell division and in particular during brain development since mRNA expression is higher at embryonic stages as compared to adult (Grisar et al., 2006). We very recently demonstrated that in fact EFHC1 is a microtubule-associated protein (MAP) playing a key role in neuronal migration (de Nijs et al., 2009). Some recent studies however showed a restricted localization of the protein at the ependymal epithelium (Suzuki et al., 2008; Conte et al., 2009) questioning its expression in brain tissue, neuronal or glial cells.

In front of these apparent controversial data, it was important to revisit the presence of this protein in different brain structures in adulthood and at a particular embryonic age.

\section{Methods}

All experiments were carried out in accordance with the directives of the European Community Council under the supervision of the Animal Care and Use Committee of the University of Liège. All efforts were made to minimize the number of animals used and their suffering.

\section{RNA extraction}

After sacrifice of NMRI mice, brains were rapidly removed, immediately frozen on dry ice and stored at $-80^{\circ} \mathrm{C}$. Coronary sections, $150 \mu \mathrm{m}$ thick, were cut on a cryostat. Samples were punched out (eight punches: one punch/section/brain region) into the appropriate locations using 18 Gauge needles and ejected into ice-chilled $1.5 \mathrm{ml}$ tubes containing $50 \mu \mathrm{l}$ of Instapur (Eurogentec, Seraing, Belgium). For cultured cells, culture medium was removed and, $1 \mathrm{ml}$ of Instapur $/ 10^{7}$ cells was directly added. Total RNA was extracted following the manufacturer's instructions. Nissl staining was performed on the sections to control for correct sample collection.

\section{RT-PCR}

Total RNA $(5 \mu \mathrm{g})$ was reverse-transcribed into CDNA in a final volume of $20 \mu \mathrm{l}$ using $2 \mu \mathrm{g}$ of random primers (Promega, Madison, WI, USA) and $200 \mathrm{U}$ of M-MLV (Promega) as described by the manufacturer. For PCR, $1 \mu \mathrm{l}$ of the resulting CDNA was submitted to 30 PCR cycles of denaturation at $94^{\circ} \mathrm{C}$ for $30 \mathrm{~s}$, annealing at $60^{\circ} \mathrm{C}$ for $30 \mathrm{~s}$ and extension at $72^{\circ} \mathrm{C}$ for $30 \mathrm{~s}$ in a total volume of $50 \mu \mathrm{l}$ of PCR reaction mixture containing (final concentration): "Green Go Taq PCR Buffer" $1 \times$ (Promega), $200 \mu \mathrm{m}$ dNTPs, $0.5 \mu \mathrm{M}$ of each primer (Forward: 5'-CTGGGCAGGACTAAAGTTGTT-3', exon8; Reverse: 5' GCTTCTGGATCCGGTTCTGAA-3', exon9) (Eurogentec) and $5 \mathrm{U}$ of Go Taq polymerase (Promega).

\section{Immunohistochemistry}

Adult NMRI mice were anesthetized by injection of Nembutal $(0.6 \mathrm{mg} / 10 \mathrm{~g}$ of body weight) and perfused transcardially with $0.9 \%$ $\mathrm{NaCl}$ followed by $4 \%$ paraformaldehyde (PFA, Sigma, St. Louis, MO, USA) dissolved in $130 \mathrm{mM} \mathrm{Na}$-phosphate buffer, $\mathrm{pH}$ 7.4. Brains were removed from the skull, postfixed in the same fixative for $1 \mathrm{~h}$ and immersed overnight at $4{ }^{\circ} \mathrm{C}$ in $30 \%$ sucrose solution in phosphatebuffered saline (PBS; $140 \mathrm{mM} \mathrm{NaCl}, 2.7 \mathrm{mM} \mathrm{KCl}, 1.5 \mathrm{mM} \mathrm{KH} 2 \mathrm{PO}_{4}$, $8.1 \mathrm{mM} \mathrm{Na}_{2} \mathrm{HPO}_{4}, \mathrm{pH} 7.4$ ). Once frozen, brains were cut into $20 \mu \mathrm{m}$ sections using a cryostat. Free-flotting sections were collected in antifreeze solution (10× PBS containing $0.5 \%$ polyvinylpyrrolidone, $15 \%$ ethylene glycol and $15 \%$ sucrose) and were stored at $-20^{\circ} \mathrm{C}$ 
until use. Brains from E16 embryos were fixed in $4 \%$ paraformaldehyde for $6 \mathrm{~h}$ and incubated overnight in PBS containing $30 \%$ sucrose. They were frozen, cut into $14 \mu \mathrm{m}$ sections using a cryostat and finally mounted onto polylysine-coated glass slides. For immunohistochemical studies, sections were washed 3 times for $10 \mathrm{~min}$ each in PBS and were incubated with $0.6 \% \mathrm{H}_{2} \mathrm{O}_{2}$ in PBS for 20 min. After three washes in PBS, sections were incubated in PBS containing $0.3 \%$ Triton X-100 (Sigma) and 10\% normal goat serum (NGS, Sigma) for $2 \mathrm{~h}$ at room temperature. Rabbit polyclonal anti-EFHC1 antibody (lkeda et al., 2005) diluted 1:50 in PBS containing $0.1 \%$ Triton X-100 and 5\% NGS was added to sections and incubated $48 \mathrm{~h}$ at $4{ }^{\circ} \mathrm{C}$. Following three washes in PBS $(10 \mathrm{~min}$ each), sections were incubated $1 \mathrm{~h}$ at room temperature with biotinylated goat anti-rabbit IgG (Vector Laboratories, Burlingame, CA, USA) diluted $1: 1000$ in PBS containing $0.1 \%$ Triton $X-100$ and $2 \%$ mouse serum (Sigma). After three additional washes in PBS, sections were incubated with streptavidin-biotin-peroxydase solution prepared using the $A B C$ kit (Vector Laboratories). The immunoperoxidase staining was revealed for $6 \mathrm{~min}$ in a $0.05 \%$ solution of $3,3^{\prime}$-diaminobenzidine (DAB, Sigma) to which $0.005 \%$ hydrogen peroxide was added. The reaction was stopped by three washes in PBS, sections were mounted on slides, air-dried and coverslipped. To test again the specificity, the anti-EFHC1 antibody was preincubated with a 20 fold molar excess of the purified antigen (peptide corresponding to residues $554-639$ of EFHC1 protein). This has also been used several times previously using whole brain extracts followed by western blotting.

\section{Immunofluorescence}

\section{Tissue sections}

Brain sections, prepared following the same procedure as for immunohistochemistry, were washed 3 times for $10 \mathrm{~min}$ each in PBS and incubated for $2 \mathrm{~h}$ at room temperature in PBS containing $0.3 \%$ Triton X-100 and non-fat dry milk $(30 \mathrm{mg} / \mathrm{ml})$. Primary antibodies were then added in PBS containing $0.1 \%$ Triton X-100 and non-fat dry milk $(15 \mathrm{mg} / \mathrm{ml})$ and incubated $48 \mathrm{~h}$ at $4{ }^{\circ} \mathrm{C}$. After three washes in PBS, fluorescently labeled secondary antibodies were added in PBS containing $0.1 \%$ Triton X-100 and non-fat dry milk $(15 \mathrm{mg} / \mathrm{ml})$ for $3 \mathrm{~h}$ at room temperature. The same washing procedure was repeated. Cell nuclei were labeled by incubation for 5 min with TOPRO-3 (Molecular Probes, Eugene, OR, USA) diluted 1:500 in PBS. Preparations were mounted in Vectashield Hard Set Medium or in Vesctashield Hard Set Medium with DAPI (Vector Laboratories).

\section{Cell cultures}

Cells were grown on glass coverslips coated with polyornithine $(0.1 \mathrm{mg} / \mathrm{ml})$ (Sigma) for $1 \mathrm{~h}$ at room temperature and laminin $\left(5 \mu \mathrm{g} / \mathrm{ml}\right.$ ) (MP Biomedicals, Santa Ana, CA, USA) for $2 \mathrm{~h}$ at $37^{\circ} \mathrm{C}$. They were fixed with $4 \%$ paraformaldehyde in PBS for $15 \mathrm{~min}$ at room temperature. After three washes in PBS (10 min each), cells were permeabilized with $0.1 \%$ Triton X-100 in PBS for $10 \mathrm{~min}$ and once again washed 3 times in PBS (10 min each).

The same protocol as for tissue sections was applied with minor modifications. Cells were incubated for $1 \mathrm{~h}$ in reconstituted blocking solution (non-fat dry milk $(15 \mathrm{mg} / \mathrm{ml})$ in PBS). The primary and secondary antibodies were added to cells in the blocking solution and the incubation period with the primary antibody was reduced to $18 \mathrm{~h}$. The preparations were mounted in Vectashield Hard Set Medium with DAPI (Vector Laboratories).

Immunofluorescent tissue sections and cells preparations were observed with a Zeiss Axiovert 135 microscope coupled to a laser scanning confocal system (MCR-1024, Bio-Rad) equipped with a filter system for fluorescein isothiocyanate (FITC, absorption: $490 \mathrm{~nm}$ and emission: $520 \mathrm{~nm}$ ) and rhodamine Red-X (RRX, absorption: $570 \mathrm{~nm}$ and emission: $590 \mathrm{~nm}$ ). Objectives $20 \times, 40 \times$ or $63 \times$ with zoom $1.5 \times$ were used, $2 \times$ or $3 \times$ accumulations were made and Z-series were realized. Single channel collection has been made. For immunohistochemistry, sections were observed with a Nikon TE 2000-U microscope coupled to a digital camera DXM1200F. Sections were viewed with a $20 \times$ or a $40 \times$ objective (with 1.5 zoom) and captured with exposure time, brightness, and contrast being held constant. Images were processed using Image J software and Photoshop software (Adobe Systems).

Among primary antibodies used for tissue sections and cells cultures, rabbit anti-EFHC1 (Ikeda et al., 2005) polyclonal antibody was used at 1:50 dilution, mouse anti-neuronal nuclei (NeuN, Chemicon, Temecula, CA, USA) monoclonal antibody was used at 1:250 dilution, mouse anti-glial fibrillary acidic protein (GFAP, Sigma) monoclonal antibody was used at 1:400 dilution, mouse anti-alphatubulin (clone B-5-1-2, Sigma) monoclonal antibody was used at 1:2000 dilution, mouse anti-nestin (Chemicon) monoclonal antibody was used at 1:100 dilution, mouse anti-Tuj1 (Covance) monoclonal antibody was used at 1:1000 dilution and mouse anti-brain lipidbinding protein (BLBP, Chemicon) monoclonal antibody was used at $1: 1000$ dilution.

Among secondary antibodies, rhodamine Red-X (RRX)conjugated donkey anti-rabbit IgG, fluorescein isothiocyanate (FITC)-conjugated donkey anti-rabbit IgG, RRX-conjugated donkey anti-mouse IgG and FITC-conjugated donkey anti-mouse IgG were used at 1:600 dilution (all from Jackson Immunoresearch Laboratories, West Grove, PA, USA). Controls experiments consisted of omitting the primary or the secondary antibodies from the pertinent solution and, for EFHC1, by preincubating the antibody with 20 -fold molar excess of purified immunogenic peptide.

\section{Cells cultures and cell cycle synchronization}

Neural stem cells (NSC) were obtained from E16 mice embryos using a previously described procedure (Ben-Hur et al., 1998). Brains were freshly dissected, striata were excised and collected in PBS supplemented with $4.5 \mathrm{~g} / \mathrm{l}$ glucose (PBSG). Isolated striata were then washed in PBSG and resuspended in DMEM-F12 medium (GIBCO, Invitrogen, Merelbeke, Belgium) containing N2 and B27 supplements (GIBCO) and $20 \mathrm{ng} / \mathrm{ml}$ epidermal growth factor (PeproTech, London, UK). Afterwards, they were dissociated by passing through a firepolished Pasteur pipette. The resulting cell suspension was filtered through a $15 \mu \mathrm{m}$ nylon sieve. Five milliliter of cell suspension at a concentration of $5 \times 10^{4}$ cells $/ \mathrm{ml}$ were transferred to non-adherent T25 culture flask (Greiner Bio-One, Frickenhausen, Germany). Half of the medium was renewed every 4 days.

The mouse neuroblastoma N2A cells were maintained in Dulbecco's modified Eagle's medium (DMEM) (GIBCO) supplemented with $5 \%$ fetal bovine serum (FBS) (Greiner Bio-one). Cells were grown at $37^{\circ} \mathrm{C}$ in a humidified incubator with a $5 \% \mathrm{CO}_{2}$ atmosphere.

Cells were synchronized at the $\mathrm{G} 1 / \mathrm{S}$ boundary by a double thymidine block and release protocol (Merril, 1998). Briefly, exponentially growing cells were treated with $2 \mathrm{mM}$ thymidine (Sigma) for $16 \mathrm{~h}$, washed twice with DMEM and maintained in fresh medium containing $24 \mu \mathrm{M}$ deoxycytidine (Sigma) for $9 \mathrm{~h}$. Cells were blocked again with $2 \mathrm{mM}$ thymidine for $16 \mathrm{~h}$, followed by a release in fresh medium containing $24 \mu \mathrm{M}$ deoxycytidine for $8 \mathrm{~h}$.

For culture of radial glial cells, cerebella obtained from E16 mouse embryos were minced with $0.25 \%$ trypsin and $0.01 \%$ DNase 1 (Sigma) for $22 \mathrm{~min}$ at $37{ }^{\circ} \mathrm{C}$. After trituration by a flamed Pasteur pipette and filtration through a $40 \mathrm{~mm}$ nylon sieve, cells were seeded as $50 \mu \mathrm{l}$ drops containing 125,000 cells on glass coverslips coated with $0.1 \mathrm{mg} / \mathrm{ml}$ polyornithine. After $30 \mathrm{~min}$, cells were washed once with cold PBS to remove loosely adhering neurons and growth medium (MEM (GIBCO) containing $6 \mathrm{~g} / \mathrm{l}$ glucose and $10 \%$ horse serum (GIBCO)) was added. This medium was changed every 2 days for 6 days. 
(A)
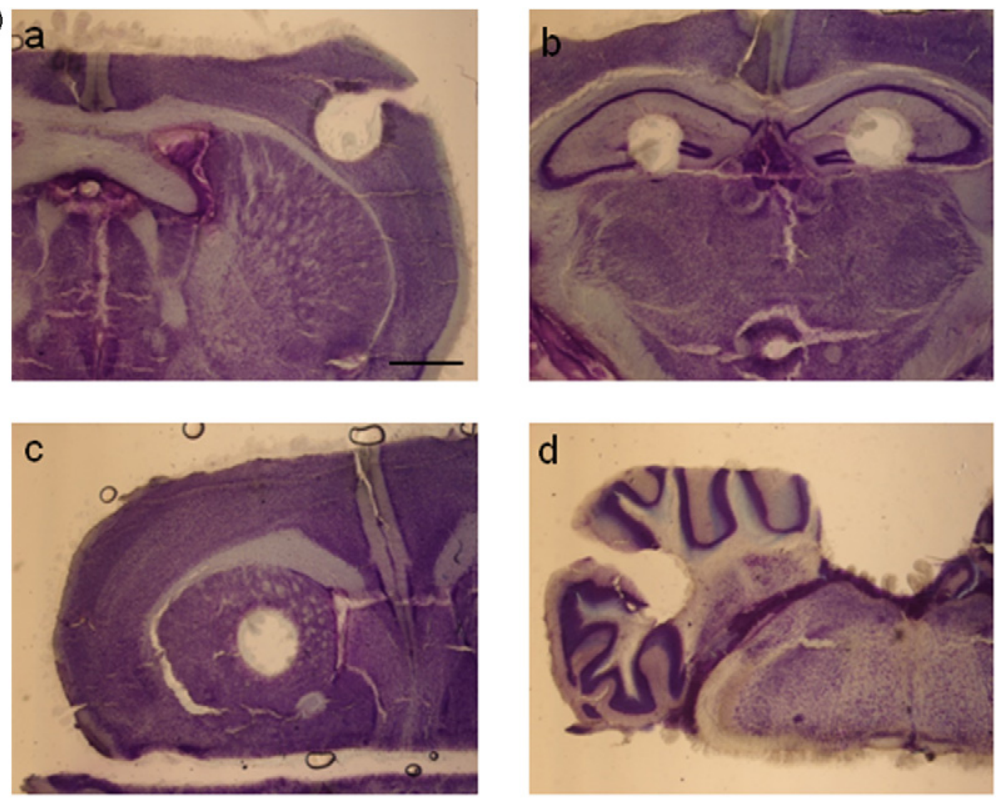

(B)

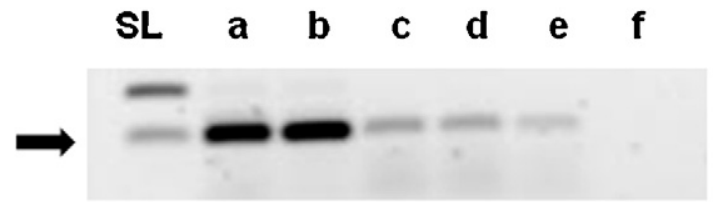

(C)

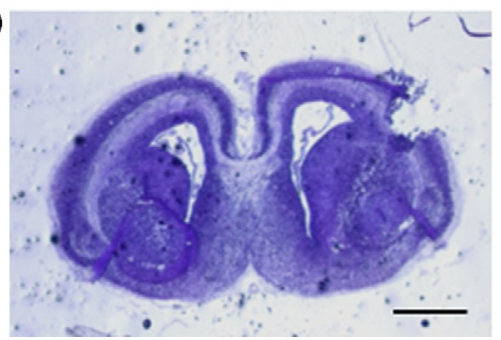

(D)

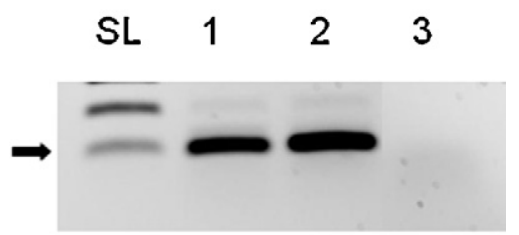

Figure 1 EFHC1 mRNA expression in adult and E16 mouse brain. (A) Nissl coloration of adult mouse brain sections punched in different areas: (a) cortex, (b) hippocampus, (c) striatum and (d) cerebellum. Scale bar: $1800 \mu \mathrm{m}$. (B) EFHC1 RT-PCR on mRNA extracts from (a) cortex, (b) entire coronal section, (c) hippocampus, (d) striatum and (e) cerebellum of adult mouse brain. The arrow indicates the band corresponding to the EFHC1 transcript. A control without mRNA is shown in (f). (C) Nissl coloration of E16 mouse brain section punched in cortex. (D) EFHC1 RT-PCR on mRNA extracts from the biopsy shown in C (1) and an entire coronal section (2) of E16 mouse brain. The arrow indicates the band corresponding to the EFHC1 transcript. Control without mRNA is shown in 3. Scale bar: $900 \mu \mathrm{m}$.

\section{Results}

\section{EFHC1 mRNA is detected in several brain structures of adult and E16 mice embryos}

To verify the expression of mRNA encoding EFHC1 in adult mouse brain, we first performed RT-PCR analysis on RNA samples isolated from not only whole brain slices (Fig. 1Bb) but also from biopsies punched from the neocortex, hippocampus, striatum and cerebellum (Fig. $1 \mathrm{Aa}-\mathrm{d}$ ) that do not contain either ependymal tissue or meninges. As shown in Fig. 1B, EFHC1 transcript was expressed in all the ana- lyzed areas. Note that the expression was more intense in the whole brain and in the cortex than in other structures.

By quantitative RT-PCR, we have previously observed that EFHC1 mRNA expression was 2-10 times higher at embryonic stages than in neonates and adults (Grisar et al., 2006). Previous works using in situ hybridization (Suzuki et al., 2008; Conte et al., 2009) have shown limited expression of the EFHC1 transcript at the ventricular borders. We used an alternative method: RNA samples were extracted from punched biopsies of cortical zones or from whole brain sections of E16 embryos (Fig. 1C) and were analyzed by RTPCR. As shown in Fig. 1D, a specific band was observed in both cases. This shows that also in embryos EFHC1 mRNA 
(A)
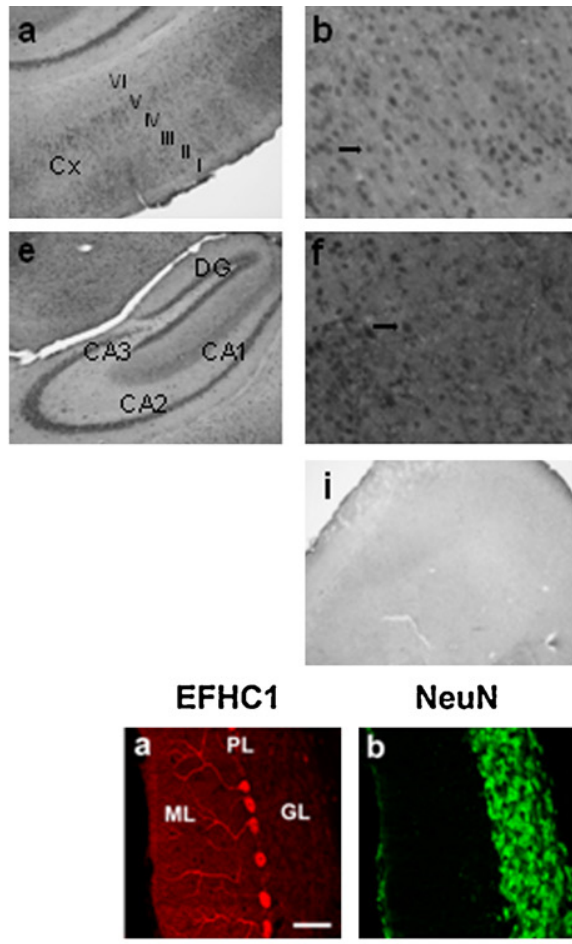

(C)

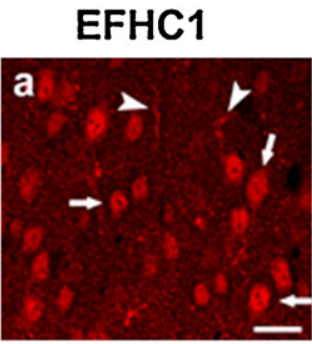

EFHC1

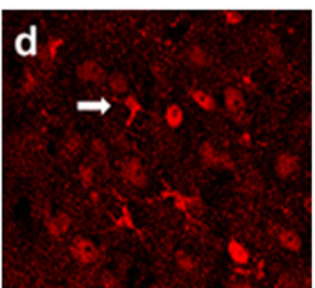

(D)

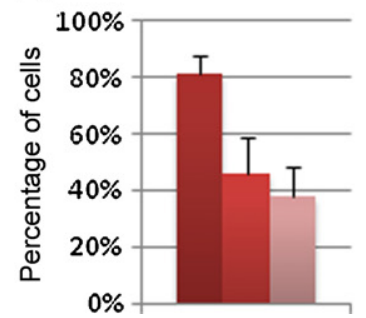

neuN+ cells

- EFHCl+cells

NeuN+/EFHCl+
cells
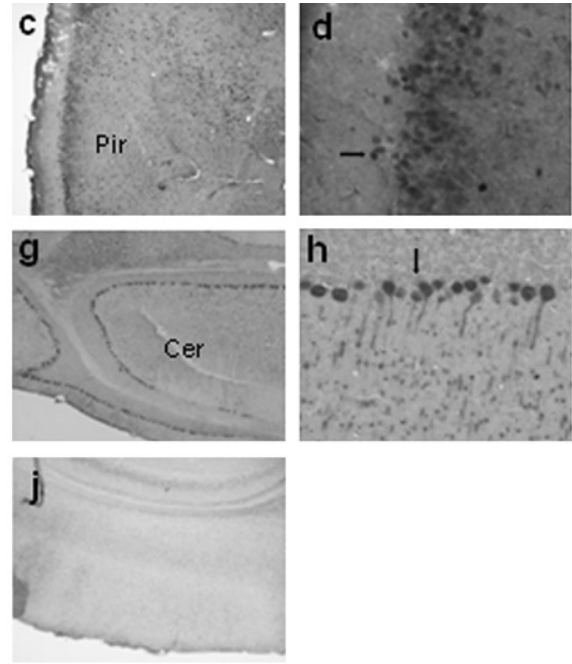

Merged
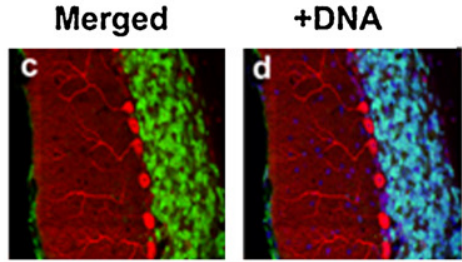

NeuN + DNA

Merged
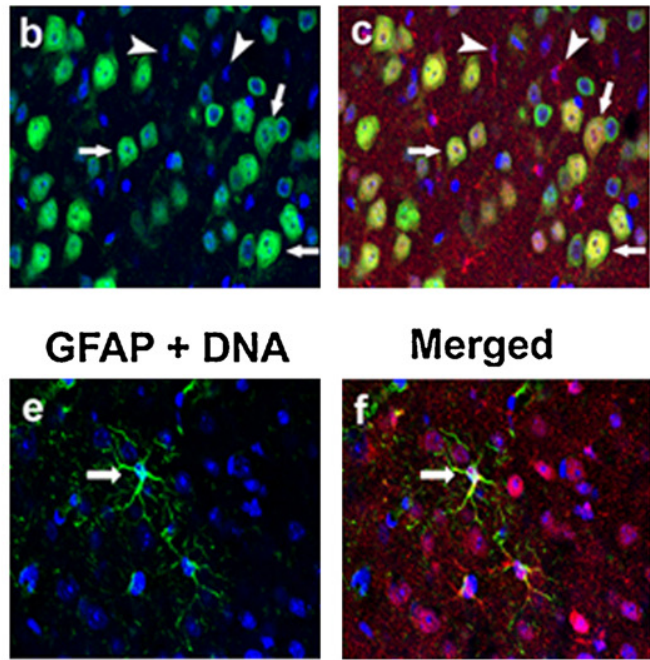

\section{Merged}

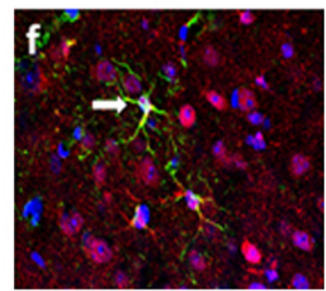

(E)

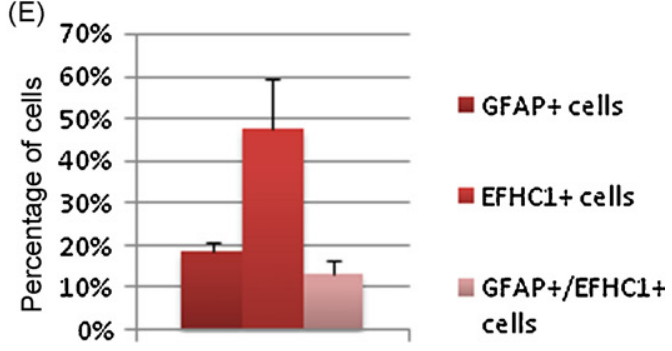

Figure 2 Localization of EFHC1 in adult mouse brain. (A) Coronal sections were labeled using anti-EFHC1 antibody. Different brain areas are shown: (a, b, i, j) neocortex, (c, d) piriform cortex, (e, f) hippocampus, (g, h) cerebellum. Enlargement of the cortical layer $\mathrm{V}(\mathrm{b})$, of the piriform cortex (d), of the hippocampus (f) and of the cerebellum (h) with arrows pointing on cells exhibiting significant staining in the soma. Control sections without primary antibody (i) or with addition of anti-EFHC1 antibody adsorbed with immunizing peptide (j). Cx: cortex; I, II, III, IV, V, VI: cortical layers; DG: dentate gyrus; Pir: piriform cortex; Cer: cerebellum. Scale bar: $400 \mu \mathrm{m}$ for a, c, e, g, i, j and $80 \mu \mathrm{m}$ for b, d, f, h. (B) EFHC1 localization in Purkinje cells in adult cerebellum. Double immunofluorescent labeling experiment with anti-EFHC1 (a, c, d, in red) and anti-NeuN (b, c, d, in green) antibodies. DNA was 
expression is likely not restricted to cells lining the cerebral ventricles.

\section{EFHC1 protein is detected in brain of E16 mice embryos and adult mice in neuronal and glial cells}

The distribution and cellular localization of EFHC1 in adult mouse brain were then studied by immunohistochemistry using a previously well validated anti-EFHC1 antibody (Ikeda et al., 2005; de Nijs et al., 2006). In the adult mouse neocortex, the labeling was present in cells of all cortical layers (Fig. 2Aa) particularly in cells resembling pyramidal neurons in cortical layer $\mathrm{V}$ (Fig. 2Ab) and in the piriform cortex (Fig. 2Ac, d). Within the hippocampus, neuronal cells in the $C A$ and the dentate gyrus (DG) regions were stained (Fig. 2Ae, f). Expression was not restricted to the pyramidal cells layer but was also observed in cells located in the striatum oriens, radiatum and lacunosum moleculare. At higher magnification, the protein appeared evenly distributed in the cytoplasm (Fig. 2Ab, d, f, arrows). A labeling was also clearly observed in Purkinje cells of the cerebellum (Fig. 2Ag, h, arrow) and, in particular, the first order apical dendrites. In all these experimental conditions, the immunostaining disappeared or was considerably attenuated after preabsorption of the antibody with purified immunizing peptide (Fig. 2Ai, j).

EFHC1 immunofluorescence was in particular clearly observed in cerebellar Purkinje cells with a labeling of the soma, the nucleus and the dendritic arborizations extending throughout the molecular layer (Fig. 2B). A faint signal was also present in the molecular and granule cell layers. The last one was identified by co-labeling using anti-NeuN antibody (Fig. 2B).

Double immunofluorescence was performed in order to specify the nature of EFHC1-expressing cells in the adult mouse cortex. Counting the number of double labeled cells indicates that the majority $(78 \%)$ of EFHC1 positive cells express NeuN and so correspond to neurons (Fig. 2Ca-c, arrows; Fig. 2D). There were also $20 \%$ of EFHC1 positive cells that express GFAP and thus correspond to astrocytes (Fig. 2Cd-f, arrows; Fig. 2E). Note that the labeling was also present in the proximal portion of astrocytic processes.

Another way to illustrate the eventual expression of this gene by neural structures is the use of nerve cell lines in culture. We extensively used this approach and Fig. 3D shows an example of clear synthesis of EFHC1 by mouse neuroblastoma cells (N2A cells), as already illustrated in the past with HEK-293, HeLa and COS cell lines (de Nijs et al., 2006). Again in neuronal cells in culture, EFHC1 clearly sticks to the mitotic spindle and the midbody during cellular mitosis (Fig. 3Da-f).
Furthermore, in order to establish how early, in culture the EFHC1 gene was expressed, we investigated its presence in neural stem cells (NSC). These NSC were prepared from embryonic mice striata, as previously described (Bez et al., 2003). They were allowed to form neurospheres. First, the expression of EFHC1 transcript was confirmed by RT-PCR (Fig. 3A). Then, double immunofluorescent labeling was performed using anti-nestin, a neural stem cells marker, and anti-EFHC1 antibodies. EFHC1 labeling was present in all cells composing neurospheres (Fig. 3B). During mitosis, labeling was present all over the cytoplasm and was strongly concentrated at the mitotic spindle (Fig. $3 \mathrm{Ca}-\mathrm{C}$ ) and the midbody (Fig. 3Cd-f).

Putting together, these results clearly demonstrate the ability of neuronal cells both in vivo and in vitro to synthesize EFHC1 from the earliest stages of development.

\section{Confirmation that EFHC1 is located in ependymal cells}

Using immunofluorescent experiments, a clear EFHC1 labeling was observed at the border of the ventricles and the choroid plexus lined with ciliated ependymal cells (Fig. 4). The labeling was visualized in the nucleus and the cytoplasm, including the ciliated apical surface of the cells (Fig. 4a, arrows and d). Using anti-alpha-tubulin labeling to visualize ciliary axonemes, we could observed a colocalization of EFHC1 and alpha-tubulin at the apical surface of ependymal cells (Fig. $4 \mathrm{a}-\mathrm{C}$, arrows). These results confirm the presence of EFHC1 in the ependymal cilia in adult mouse brain

\section{EFHC1 is expressed in E16 mouse brain co-localizing with radial glial fibers}

In the embryonic mouse brain at E16, the EFHC1 immunostaining was only faintly observed throughout the whole nervous tissue. In the neocortex, the labeling was poorly but clearly detectable in the cortical plate (Fig. 5a, b). The piriform cortex was also labeled (Fig. 5c, d). A particularly good signal was observed in cells lining the third ventricle (Fig. $5 \mathrm{~g}$, h), and even stronger in cells of the choroid plexus (Fig. 5e, f). Again, these signals were blocked by preincubation of the antibody with purified immunizing peptide (Fig. $5 i, j$ ). When looking more carefully the immunostaining appears mostly in structures drawing a radial organization in the cortical plate (Fig. 5a, b) and also on the border of ventricles (Fig. 5e, g). In some cells in the deep layers of the cortical plate (Fig. 5b, arrow), in the piriform cortex (Fig. $5 \mathrm{~d}$, arrow) and in the choroid plexus (Fig. 5f, arrow), the signal appeared also in the nucleus.

stained with TOPRO-3 (d, in blue). ML: molecular layer, PL: Purkinje cells layer, GL: granule cells layer. Confocal images. Scale bar: $40 \mu \mathrm{m}$. (C) Double immunofluorescent labeling using anti-EFHC1 ( $a, c, d, f$, in red) and anti-NeuN (b, c, in green) or anti-GFAP (e, f, in green) antibodies. In $(\mathrm{a}-\mathrm{C})$ : examples of NeuN-positive neurons expressing EFHC1 are indicated by arrows, arrowheads show EFHC1-labeled cells which are not expressing NeuN. In $(\mathrm{d}-\mathrm{f})$ : arrows show a GFAP-positive cell expressing EFHC1. DNA was stained with DAPI (in blue). The corresponding merged images are shown in $\mathrm{c}$ and f. Confocal images. Scale bar: $40 \mu \mathrm{m}$. (D) Quantification of $\mathrm{NeuN}^{+}$cells, EFHC1 $1^{+}$cells and $\mathrm{NeuN}^{+} / \mathrm{EFHC1}^{+}$cells. (E) Quantification of $\mathrm{GFAP}^{+}$cells, EFHC1 ${ }^{+}$cells and $\mathrm{GFAP}^{+} / \mathrm{EFHC1}^{+}$cells. For quantifications, 350 cells from each of three independent experiments were scored for NeuN or GFAP expression. (For interpretation of the references to color in this figure legend, the reader is referred to the web version of the article.) 


\section{(A) SL NSC Control}

(B)

EFHC1

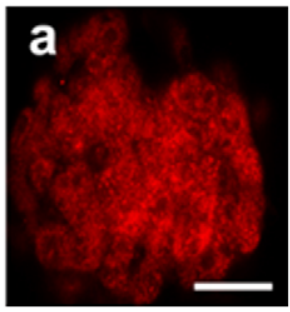

(C) EFHC1
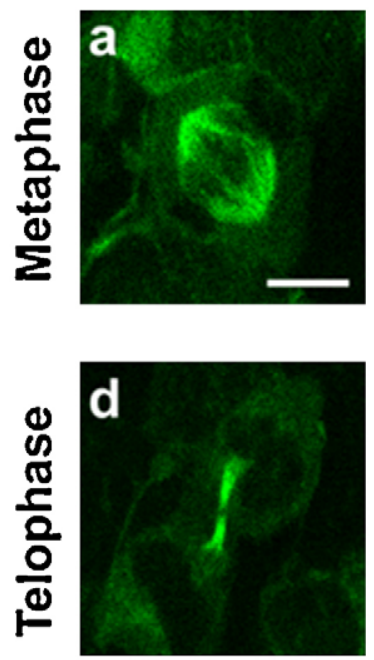

(D)
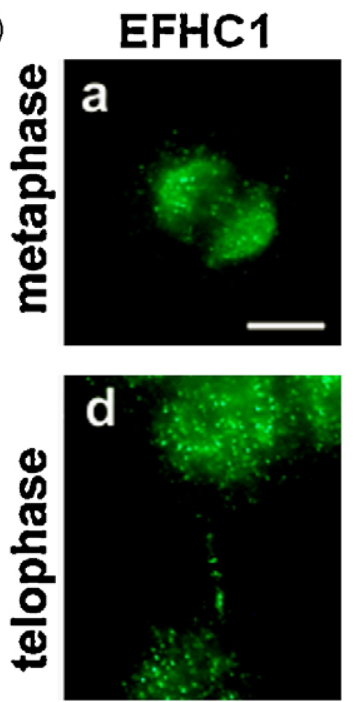
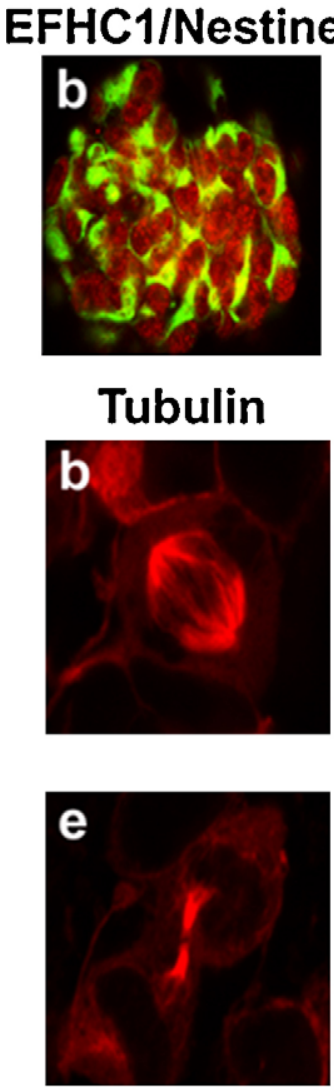

Tubulin
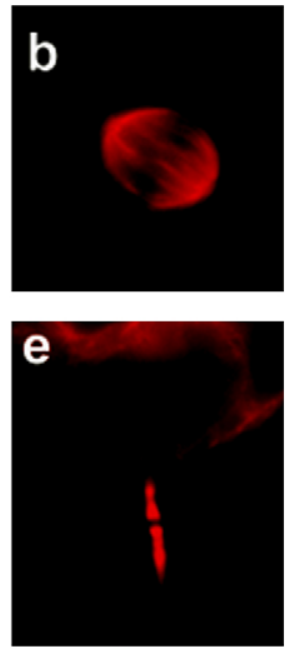

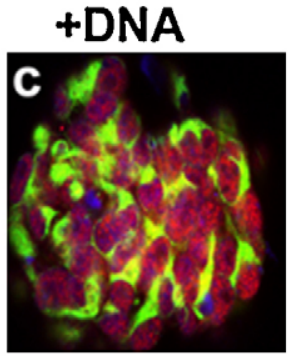

\section{Merged}
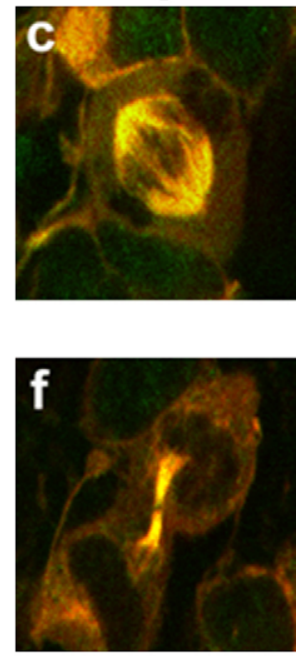

Merged
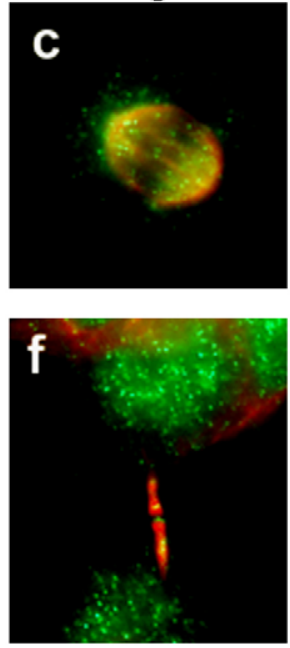

Figure 3 Association of EFHC1 with the mitotic apparatus in mouse neural stem cells and mouse neuroblastoma cells (N2A). (A) EFHC1 RT-PCR on RNA extracts from multipotent neural stem cells (NSC). Arrow indicates EFHC1 transcript. (B) Stem cells grown in neurospheres were labeled using anti-EFHC1 (in red) and anti-nestine (in green) antibodies. DNA was stained with DAPI (in blue). Scale bar: $25 \mu \mathrm{m}$. (C) Stem cells grown in neurospheres were synchronized by double thymidine block and were fixed with paraformaldehyde. Immunolabeling was realized using anti-EFHC1 (in green) and anti-alpha-tubulin (in red) antibodies. Two 

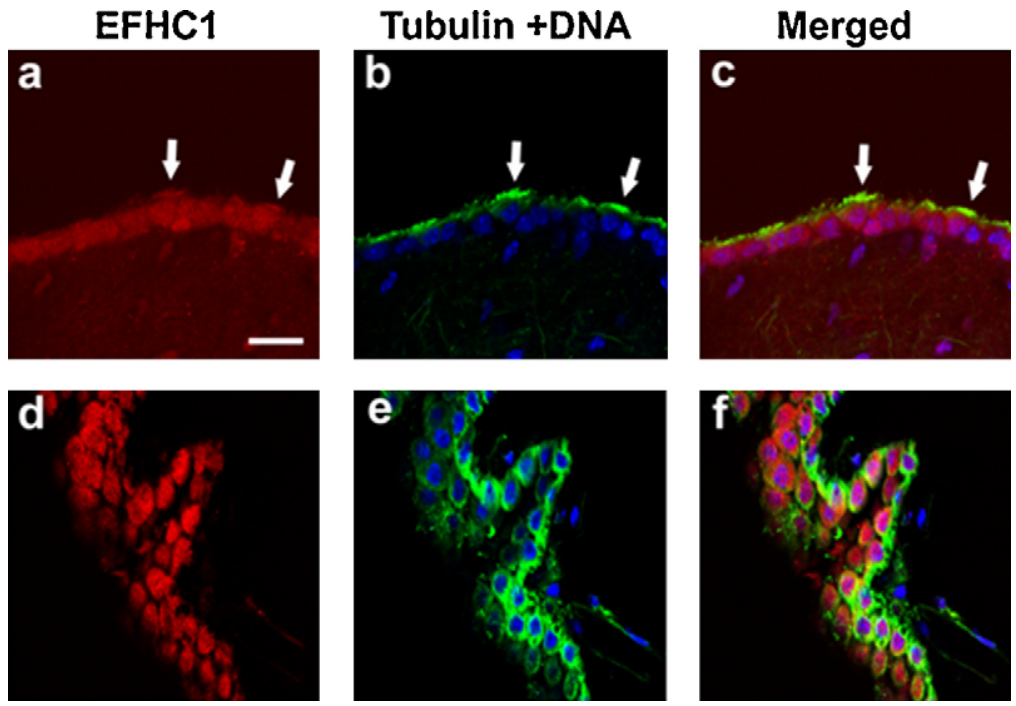

Figure 4 EFHC1 localization in ependymal cells and choroid plexus in adult mouse lateral ventricle. Double immunofluorescent labeling using anti-EFHC1 (a, d, in red) and anti-alpha-tubulin (b, e, in green) antibodies. Arrows show ependymal cells expressing EFHC1 in cilia. DNA was stained with TOPRO-3 (in blue). The corresponding merged images are shown in $\mathrm{c}$ and $\mathrm{f}$. Confocal images. Scale bar: $40 \mu \mathrm{m}$. (For interpretation of the references to color in this figure legend, the reader is referred to the web version of the article.)

This EFHC1 labeling in the cortical plate looking similar to the radial glial cell profile, we have investigated expression of EFHC1 in these cells in vitro and in vivo. As shown on the histogram (Fig. 6B), our primary culture contains $85 \%$ of radial glial cells (BLBP positive, Fig. $6 \mathrm{Aa}$ ) for only $7 \%$ of neuronal cells (Tuj1 positive, Fig. 6Ab). By performing double immunofluorescent labeling using anti-EFHC1 and anti-BLBP antibodies, we observed not only that EFHC1 is expressed in radial glia primary cultures but also co-localized with radial glia extensions in E16 embryos brain sections (Fig. 6C and D).

\section{Discussion}

The present work revisits the cellular distribution in the adult and embryonic CNS of a new gene called EFHC1 mutated in JME. Indeed, in their seminal paper, Suzuki et al. (2004) showed by northern blotting the presence of the transcript in various structures of the mice body, including brain, whereas the protein was detected in different adult brain structures and nerve cells in vitro by immunocytochemistry. Later on, however, the same authors questioned these previous results after having shown the inappropriateness of their initial antibody using EFHC1 KO mice and therefore showed a restricted localization of the protein at the ependymal epithelium (Suzuki et al., 2008). Similar results were also presented by Conte et al. (2009).

Since we have previously suggested a possible role of EFHC1 in cell division and brain development (de Nijs et al., 2006; Grisar et al., 2006) and recently confirmed that this molecule is a MAP implicated in neuronal migration (de Nijs et al., 2009), it was important, in front of these controversial initial data, to check the presence of this transcript and protein in some nerve cells in culture as well as in brain structures both in adulthood and during development.

First, we attempted to detect mRNAs encoding EFHC 1 by in situ hybridization. Our data were similar to those published by the Allen Brain Atlas (www.brain-map.org), i.e.: a poor specific signal in some structures of the adult brain (hippocampus, cerebellum), including ependymal epithelium, indicating, as already reported (Suzuki et al., 2008; Conte et al., 2009), a probable very low level of expression.

Therefore, EFHC1 mRNA expression was studied in the adult and embryonic mouse brain using RT-PCR, a very sensitive and specific technique. In order to avoid any contamination by choroid plexus or cells lining the brain ventricles, samples were punched out from brain sections so that we could check they were correctly isolated. Using this approach, we showed that, whatever the age, EFHC1 mRNA was indeed expressed, also sometimes at low level, in all structures tested i.e. cortex, striatum, hippocampus and cerebellum in adult but also cortex in $\mathrm{E} 16$ embryo. These data clearly demonstrated that EFHC1 mRNA expression is not restricted to choroid plexus or to cells lining the ventricles as suggested by Suzuki et al. (2008). We confirmed however that EFHC1 mRNA expression level was particularly strong in ependymal cells (along the ventricles) but in many other brain structures, scattered positive cells were

mitotic phases are shown: metaphase $(a-c)$ and telophase $(d-f)$. Right panels $(c, f)$ represent merges of corresponding images on left and middle panels. Confocal images. Scale bar: $10 \mu \mathrm{m}$. (D) Neuroblastoma cells (N2A) were fixed with paraformaldehyde and immunolabeled using anti-EFHC1 (in green) and anti-alpha-tubulin (in red) antibodies. Two mitotic phases are shown: metaphase (a-c) and telophase $(d-f)$. Right panels (c, f) represent merges of corresponding images on left and middle panels. Confocal images. Scale bar: $10 \mu m$. (For interpretation of the references to color in this figure legend, the reader is referred to the web version of the article.) 

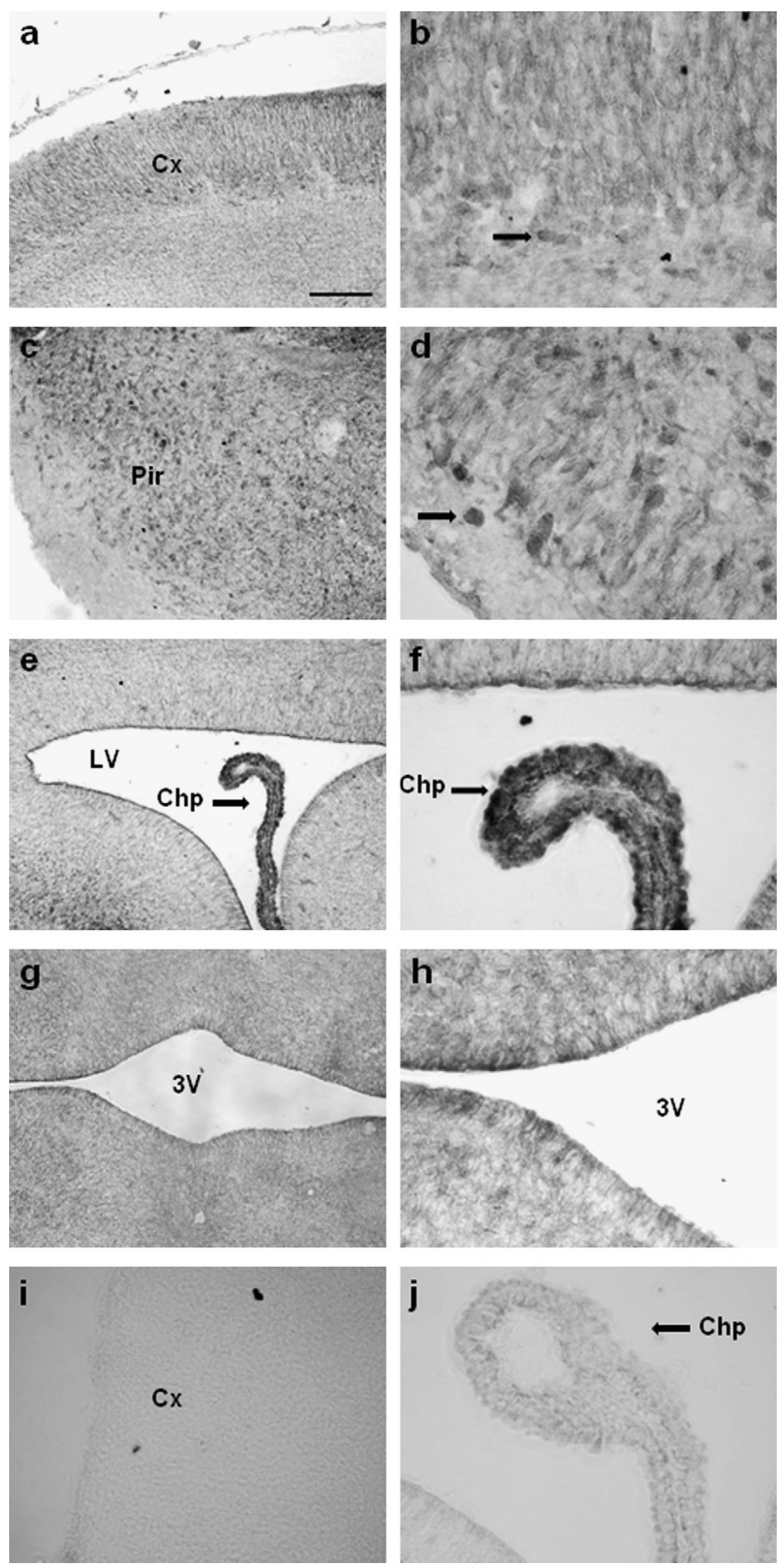

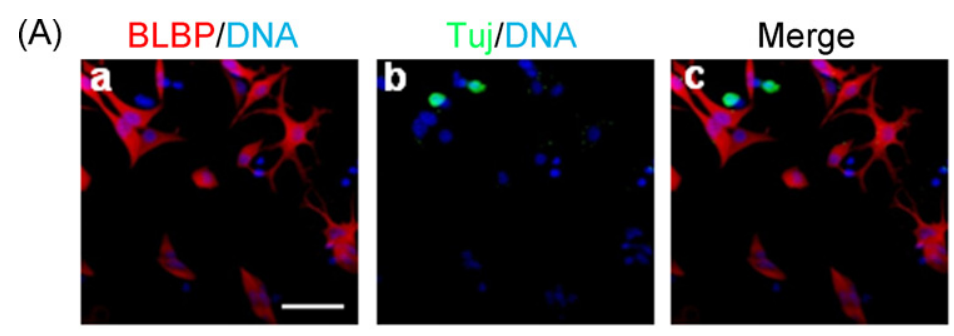

(B)

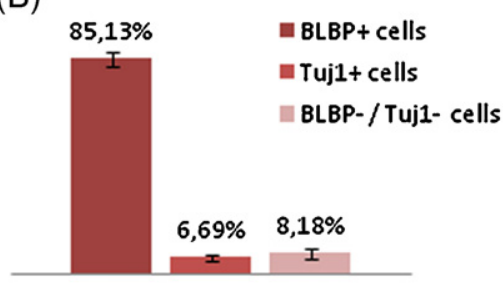

(C)

BLBP/DNA
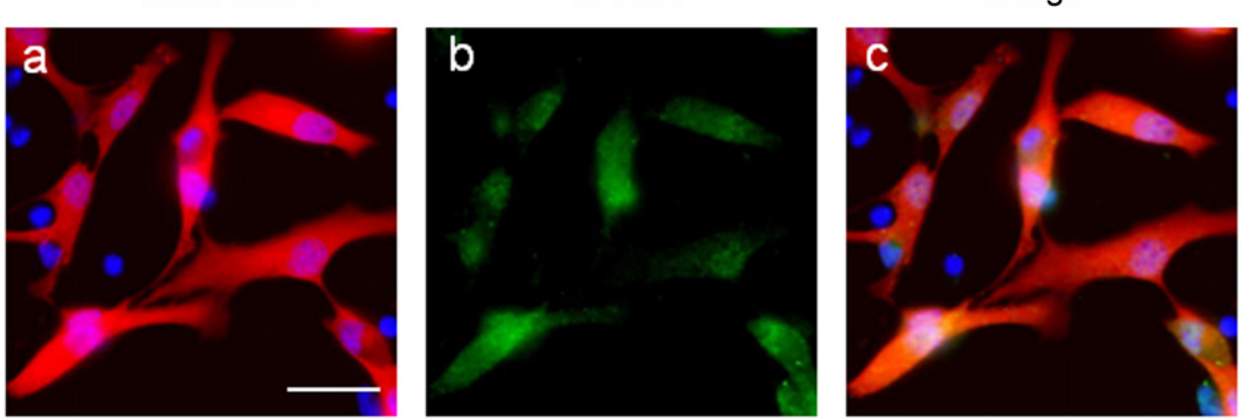

(D)

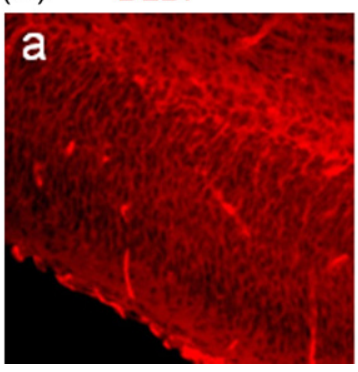

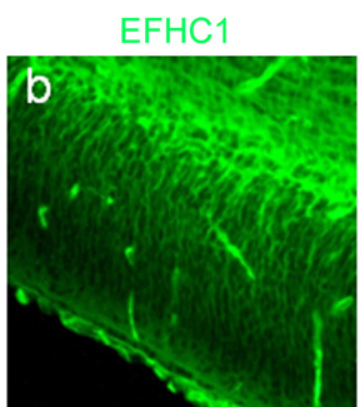

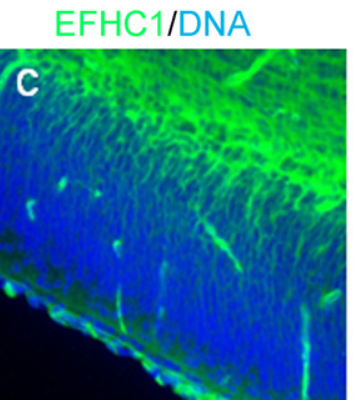

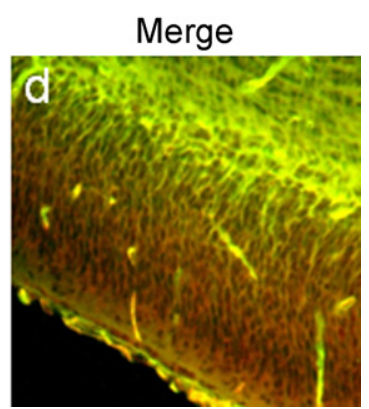

Figure 6 EFHC1 expression in radial glial cells in vitro and in embryonic mouse brain cortex (E16). (A) Double immunofluorescent labeling on radial glial cells in vitro using anti-BLBP (a, c, in red) and anti-Tuj1 (b, c, in green) antibodies. Scale bar: $80 \mu \mathrm{m}$. (B) Histogram indicating percentage of $\mathrm{BLBP}^{+}$cells and Tuj1 $1^{+}$cells in radial glial cells culture from E16 mouse embryos. For quantification, 250 cells from each of three independent primary cultures were scored for BLBP or Tuj1 expression. (C) Double immunofluorescent labeling on radial glial cells in vitro using anti-BLBP (a, c, in red) and anti-EFHC1 (b, c, in green) antibodies. Scale bar: $40 \mu \mathrm{m}$. (D) Double immunofluorescent labeling experiments on E16 mouse brain neocortex sections with anti-BLBP (a, d, in red) and anti-EFHC1 (b-d, in green) antibodies. Scale bar: $160 \mu \mathrm{m}$. (For interpretation of the references to color in this figure legend, the reader is referred to the web version of the article.)

also observed (Allen Brain Atlas, www.brain-map.org). Our results are also in agreement with those obtained by Northern blot on various human brain structures (Suzuki et al., 2004). In embryonic brain, using RT-PCR analysis on cortices samples taken at E16, we demonstrated the presence of the transcript in cells that do not line the ventricles. This it is not in agreement with the one of Suzuki et al. (2008) as they observed an expression that seems restricted to ventricles borders. However, their analysis was made at E18, an age where radial glial cells start to differentiate into ependy- mal cells (Spassky et al., 2005). This differentiation process, called ciliogenesis, requires de novo production of up to 200-300 centrioles depending on the tissue (see Dawe et al., 2007 for a review). Because we have previously showed that EFHC1 co-localizes with the centriole, it is probable that during ciliogenesis, EFHC1 mRNA level increases and so that it is easily detected by in situ hybridization. Moreover, it is not uncommon to see a protein playing an important role in the brain with a low level of mRNA expression like shown for several synaptotagmins (Mittelsteadt et al., 2009). This

Figure 5 Distribution of EFHC1 immunoreactivity in embryonic mouse brain (E16). Coronal sections were labeled using anti-EFHC1 antibody. Different brain regions are shown: $(\mathrm{a}, \mathrm{b})$ : neocortex, $(\mathrm{c}, \mathrm{d})$ : piriform cortex, $(\mathrm{e}, \mathrm{f})$ : lateral ventricle and choroid plexus, $(\mathrm{g}, \mathrm{h})$ : third ventricle. Control sections with addition of EFHC1 antibody adsorbed with immunizing peptide is shown in $\mathrm{i}$ and $\mathrm{j}$. Arrows indicate cells exhibiting staining in the soma. Cx: neocortex; Pir: piriform cortex; LV: lateral ventricle; chp: choroid plexus; $3 \mathrm{~V}$ : third ventricle. Scale bar represents $160 \mu \mathrm{m}$ for a, c, e, g, i and $40 \mu \mathrm{m}$ for b, d, f, h, j. 
probably explains the "apparent" discrepancies between the two studies.

Then, we studied the distribution of the protein in the brain using a previously validated purified polyclonal antibody against EFHC1 (lkeda et al., 2005; de Nijs et al., 2006). In agreement with the mRNA distribution, we observed protein expression in many adult brain structures. Labeling was detected in the different layers of the neocortex, in the piriform cortex, the hippocampus (CA region and dentate gyrus) and the cerebellum. At the cellular level, EFHC1 was detected both in neurons and glial cells, but also in some neurites like the first order dendrites of the Purkinje cells. Because EFHC1 has also been shown to be associated with flagella and motile cilia (lkeda et al., 2005), the latter being also present in ependymal epithelia covering the ventricular wall, we have paid a particular attention to an eventual co-localization of EFHC1 and ciliary tubulin in these cells. As shown, EFHC1 is present in ependymal cells and seems to co-localize with motile cilia labeled in parallel with alphatubulin.

We further investigated the cerebral distribution of the protein in the developing mouse brain (E16) and neural stem cells. At E16, the protein was strongly expressed at the border of the cerebral ventricles as well as in the choroid plexus. A significant, although faint, labeling was also observed in neocortex and piriform cortex. We have confirmed that in our recently published paper showing the expression of EFHC1 protein in E17 rat cortex (de Nijs et al., 2009). The poor expression of the signal in general does not allow a quantitative comparison with what has been observed in adulthood, although it is evident that the best pictures of nerve structures have been obtained with adult animals. Whether or not this means a significant maturation process of the production of EFHC1 with age cannot be solved by our actual work. At the subcellular level, antiEFHC1 antibody recognized principally a cytoplasmic material, quite radially organized. Double labeling experiments with an anti-BLBP antibody demonstrated that EFHC1 was expressed in fact in radial glial cells both in vitro and in vivo.

In conclusion, in agreement with Suzuki et al. (2008), we confirm the presence of EFHC1 in ependymal cells and, particularly in motile cilia, but our study suggests that its expression is broader. The specificity of the monoclonal antibody used by Suzuki et al. (2008) is obviously not questioned. What could be the reasons for these differences? First of all, it should be noted that both antibodies do not recognize the same epitope. The one of Suzuki recognize a small epitope located between the 2nd and 3rd DM10 domains whereas ours is directed against the $\mathrm{C}$-terminus of the protein (after the 3rd DM10 domain). It is therefore possible that the subcellular environment influences the detectability of the antigen so that only some parts of the protein are more accessible in different structures (Lorincz and Nusser, 2008). On the other hand, the sensitivity of the detection might be responsible for these apparent contradictions between the two works.

The broad expression of EFHC1 makes sense for a protein tagged as "axonemal". Tektins perhaps can be used as a good comparison as both tektin and EFHC1 are found in sarkosyl-stable protofilament ribbons isolated from Chlamydomonas flagella (Setter et al., 2006). By Northern blot, it was demonstrated that Tektin-1 mRNA is already expressed in mouse brain at E11 and by in situ hybridization, its presence is shown in choroid plexus, developing retina and olfactory epithelium also some staining is apparent in other brain structures (Norrander et al., 1998). At adulthood, a clear signal is observed along ventricles and choroid plexus, but scattered cells are detected in many brain structures (Allen Brain Atlas). Morever, mRNA encoding tektin-1 is detected in freshly isolated radial glial cells (Li et al., 2003). Finally tektin-2, which is also associated to mitotic spindles and midbody in $\mathrm{CHO}$ cells, is required for proper central spindle microtubule organization and cytokinesis completion (Durcan et al., 2008).

Taken together, our previous (de Nijs et al., 2006, 2009) and present results strongly suggest a particular function for EFHC1 in the central nervous system development. During ontogenesis of the cerebral cortex, neuronal progenitors are generated in the ventricular zone by a specialized and transient class of glial cells, the radial glial cells. Then, these neuronal progenitors migrate radially to establish their final position. This migration is also dependent on guidance provided by radial glial cells (Rakic, 1972). The expression of EFHC1 in radial glial cells in vitro and in embryonic mouse brain at E16 and its co-localization with the mitotic spindle in mitotic cells, confirms the possibility that this protein plays a role during neuronal progenitors division. On the other hand, because of its association with the centrosome (de Nijs et al., 2006), a microtubule-organizing center (MTOC), that plays an important function in neuronal movement (Hatten, 2002) and by its co-localization with radial glia, it could influence neuronal progenitors migration. Therefore, we hypothesize that microdysgenesis and increased thickness of cortical grey matter found by MRI studies of JME patients (Meencke and Janz, 1984; Woermann et al., 1999) could result from defects in neuronal migration due to disruption of proteins that regulate microtubules in radial glia, like EFHC1.

This opens the door to a rather new conceptual approach viewing some idiopathic generalized epilepsies like JME as developmental diseases instead of classical channelopathies.

\section{Acknowledgements}

We thank the Dr. Ritsu Kamiya (University of Tokyo) for kindly providing the polyclonal anti-EFHC1 antibody used in this study. This work was supported by the Fonds National de la Recherche Scientifique (F.R.S.-FNRS; FRSM 3.4565.03.F to $\mathrm{TG}$ and $\mathrm{BL}$ ) and the Léon Fredericq Foundation (to $\mathrm{CL}$ ). $\mathrm{CL}$ is fellow of the Fonds pour la Recherche dans l'Industrie et l'Agriculture (FRIA). BL is a research associate at the F.R.S.FNRS.

\section{References}

Ben-Hur, T., Rogister, B., Murray, K., Rougon, G., Dubois-Dalcq, M., 1998. Growth and fate of PSA-NCAM+ precursors of the postnatal brain. J. Neurosci. 18, 5777-5788.

Bez, A., Corsini, E., Curti, D., Biggiogera, M., Colombo, A., Nicosia, R.F., Pagano, S.F., Parati, E.A., 2003. Neurosphere and neurosphere-forming cells: morphological and ultrastructural characterization. Brain Res. 993, 18-29. 
Conte, F.F., Ribeiro, P.A., Marchesini, R.B., Pascoal, V.D., Silva, J.M., Oliveira, A.R., Gilioli, R., Sbragia, L., Bittencourt, J.C., Lopes-Cendes, I., 2009. Expression profile and distribution of Efhc1 gene transcript during rodent brain development. J. Mol. Neurosci. 39, 69-77.

Dawe, H.R., Farr, H., Gull, K., 2007. Centriole/basal body morphogenesis and migration during ciliogenesis in animal cells. J. Cell Sci. 120, 7-15.

de Nijs, L., Lakaye, B., Coumans, B., Léon, C., Ikeda, T., DelgadoEscueta, A.V., Grisar, T., Chanas, G., 2006. EFHC1, a protein mutated in juvenile myoclonic epilepsy, associates with the mitotic spindle through its $\mathrm{N}$-terminus. Exp. Cell Res. 312, 2872-2879.

de Nijs, L., Léon, C., Nguyen, L., Loturco, J.J., Delgado-Escueta, A.V., Grisar, T., Lakaye, B., 2009. EFHC1 interacts with microtubules to regulate cell division and cortical development. Nat. Neurosci. 12, 1266-1274.

Durcan, T.M., Halpin, E.S., Rao, T., Collins, N.S., Tribble, E.K., Hornick, J.E., Hinchcliffe, E.H., 2008. Tektin2 is required for central spindle microtubule organization and the completion of cytokinesis. J. Cell Biol. 181, 595-603.

Grisar, T., de Nijs, L., Chanas, G., Léon, C., Coumans, B., Foidart, A., Lakaye, B., 2006. Some genetic and biochemical aspects of myoclonus. Neurophysiol. Clin. 36, 271-279.

Hatten, M.E., 2002. New directions in neuronal migration. Science 297, 1660-1663.

Ikeda, T., Ikeda, K., Enomoto, M., Park, M.K., Hirono, M., Kamiya, R., 2005. The mouse ortholog of EFHC1 implicated in juvenile myoclonic epilepsy is an axonemal protein widely conserved among organisms with motile cilia and flagella. FEBS Lett. 579, 819-822.

Li, H., Berlin, Y., Hart, R.P., Grumet, M., 2003. Microtubules are critical for radial glial morphology: possible regulation by MAPs and MARKs. Glia 44, 37-46.

Lorincz, A., Nusser, Z., 2008. Specificity of immunoreactions: the importance of testing specificity in each method. J. Neurosci. 28, 9083-9086.

Medina, M.T., Suzuki, T., Alonso, M.E., Durón, R.M., MartínezJuárez, I.E., Bailey, J.N., Bai, D., Inoue, Y., Yoshimura, I., Kaneko, S., Montoya, M.C., Ochoa, A., Prado, A.J., Tanaka, M., Machado-Salas, J., Fujimoto, S., Ito, M., Hamano, S., Sugita, K., Ueda, Y., Osawa, M., Oguni, H., Rubio-Donnadieu,
F., Yamakawa, K., Delgado-Escueta, A.V., 2008. Novel mutations in Myoclonin 1/EFHC1 in sporadic and familial juvenile myoclonic epilepsy. Neurology 70, 2137-2144.

Meencke, H.J., Janz, D., 1984. Neuropathological findings in primary generalized epilepsy: a study of eight cases. Epilepsia 25, 8-21.

Merril, G.F., 1998. Cell synchronization. Methods Cell Biol. 57, 229-249.

Mittelsteadt, T., Seifert, G., Alvarez-Baron, E., Steinhauser, C., Becker, A.J., Schoch, S., 2009. Differential mRNA expression patterns of the synaptotagmin gene family in the rodent brain. $J$. Comp. Neurol. 512, 514-528.

Norrander, J., Larsson, M., Stahl, S., Hoog, C., Linck, R., 1998. Expression of ciliary tektins in brain and sensory development. J. Neurosci. 21, 8912-8918.

Rakic, P., 1972. Mode of cell migration to the superficial layers of fetal monkey neocortex. J. Comp. Neurol. 145, 61-84.

Setter, P.W., Malvey-Dorn, E., Steffen, W., Stephens, R.E., Link, R.W., 2006. Tektin interactions and a model for molecular functions. Exp. Cell Res. 312, 2880-2896.

Spassky, N., Merkle, F.T., Flames, N., Tramontin, A.D., GarciaVerdugo, J.M., Alvarez-Buylla, A., 2005. Adult ependymal cells are postmitotic and are derived from radial glial cells during embryogenesis. J. Neurosci. 25, 10-18.

Suzuki, T., Delgado-Escueta, A.V., Aguan, K., Alonso, M.E., Shi, J., Hara, Y., Nishida, M., Numata, T., Medina, M.T., Takeuchi, T., Morita, R., Bai, D., Ganesh, S., Sugimoto, Y., Inazawa, J., Bailey, J.N., Ochoa, A., Jara-Prado, A., Rasmussen, A., RamosPeek, J., Cordova, S., Rubio-Donnadieu, F., Inoue, Y., Osawa, M., Kaneko, S., Oguni, H., Mori, Y., Yamakawa, K., 2004. Mutations in EFHC1 cause juvenile myoclonic epilepsy. Nat. Genet. 36, 842-849.

Suzuki, T., Inoue, I., Yamagata, T., Morita, N., Furuichi, T., Yamakawa, K., 2008. Sequential expression of Efhc1/myoclonin1 in choroids plexus and ependymal cell cilia. Biochem. Biophys. Res. Commun. 367, 226-233.

Woermann, F.G., Free, S.L., Koepp, M.J., Sisodiya, S.M., Duncan, J.S., 1999. Abnormal cerebral structure in juvenile myoclonic epilepsy demonstrated with voxel-based analysis of MRI. Brain 122, 2101-2108.

Wolf, P., 1992. Juvenile myoclonic epilepsy. In: Epilepsy Syndrome in Infancy, Childhood and Adolescence. JL Company, pp. 313-327. 